\title{
Projections d'habitants face à des données climatiques localisées : Ruse rétrospective pour un territoire apprenant
}

Dominique Bachelart et Emmanuelle Crépeau

\section{(2) OpenEdition}

Édition électronique

URL : https://journals.openedition.org/ere/7254

DOI : $10.4000 /$ ere. 7254

ISSN : 2561-2271

Éditeur

Centr'ERE

Référence électronique

Dominique Bachelart et Emmanuelle Crépeau, «Projections d'habitants face à des données

climatiques localisées : Ruse rétrospective pour un territoire apprenant », Éducation relative à

l'environnement [En ligne], Volume 16 - 1 | 2021, mis en ligne le 10 mars 2021, consulté le 27 mai 2021.

URL : http://journals.openedition.org/ere/7254 ; DOI : https://doi.org/10.4000/ere.7254 


\title{
Projections d'habitants face à des données climatiques localisées : Ruse rétrospective pour un territoire apprenant
}

\author{
Dominique Bachelart et Emmanuelle Crépeau
}

1 La problématique du changement climatique est portée à l'attention publique des démocraties occidentales depuis de nombreuses années sans que cela ait abouti à des changements proportionnés au diagnostic sur l'évolution de l'état de la planète et de ses occupants sur les deux versants: l'épuisement des ressources et les équilibres globaux vitaux pour la poursuite de l'aventure humaine. Les scientifiques se mobilisent depuis la fin des années 1980 sur «l'effet de serre ». Les "alertes à l'humanité » se succèdent. Puisqu'on sait, pourquoi n'agit-on pas? (Dupuy, 2002). L'activité scientifique a apporté de l'information constante sur la dynamique terrestre: interaction entre l'atmosphère, les océans, les glaciers et les banquises, la biosphère océanique et continentale. Elle s'attache de façon de plus en plus précise à appréhender l'impact de l'activité humaine sur la planète et des risques qui y sont liés. La dérive anthropogénique de l'effet de serre est maintenant largement admise comme une donnée robuste. Cela n'exclue pas des postures de déni ou des démarches puissantes de lobbying climato-sceptique (Oreske, Conway, 2012).

2 «La science maintient l'exigence d'anticipation et a développé des outils de modélisation qui constituent de puissants collecteurs et opérateurs de connaissance " (Charles, 2006, p 51). D'abord traitée de manière globale avec des messages sur l'effet de serre, puis les gaz à effet de serre, sur l'empreinte écologique, le seuil de réchauffement de $2^{\circ}$, le facteur $4 . .$. l'information commence à se traiter au niveau local comme problématique à venir dans chaque territoire (Bertrand et Richard, 2014 p. 199). L'enjeu d'engagement de la population et sa participation aux orientations d'action dans le territoire est alors crucial. 
3 Cet article propose une réflexion méthodologique sur l'accompagnement et l'engagement de la population. Une première partie examine le contexte territorial de la politique climat du Parc naturel régional Loire-Anjou-Touraine (PNR LAT) et ses premières actions en direction du public. Elle présente un dispositif d'ateliers de prospective territoriale sur le climat. Enfin, elle décrit la méthodologie de recherche par observation participante en co-production avec une praticienne en réflexion sur sa pratique de médiation. La deuxième partie présente les actions de sensibilisation menées par le PNR LAT en direction de la population. En examinant différentes postures - expertise, sensibilisation, diffusion des connaissances scientifiques, coproduction de savoirs - elle interroge l'articulation entre la vision descendante de diffusion d'informations et une vision d'engagement du public dans des espaces d'échange et d'orientation sur les données scientifiques produites et les politiques à mettre en place. La troisième partie présente les résultats de l'analyse. Ceux-ci portent d'une part sur la réflexion sur posture de médiation et d'autre part sur les effets sur les participants. La quatrième partie montre l'effet rétrospectif produit par la projection sur le temps long, amenant les participants à des scénarios engageant les actions à mettre en œuvre dès à présent. En examinant l'importance des co-productions de connaissances et de récits croisés en créant les conditions d'un territoire « apprenant ».

\section{Le contexte territorial : engagement d'un PNR et actions de médiation grand public dans un plan « climat »}

4 Des territoires français se sont engagés dès 2012, dans des « plans climat, air, énergie, territoriaux », axés sur la réduction des gaz à effet de serre, sur la dépendance énergétique. Cette procédure se généralise avec le décret du 28 juin 2016 relatif à la loi sur la transition énergétique pour la croissance verte qui confie en l'élaboration et la mise aux intercommunalités de plus de 20000 habitants à des échelons représentatifs des enjeux de mobilité (bassin de vie) et d'activité (bassin d'emploi). Les objectifs d'atténuation sont alors associés de manière plus explicite aux objectifs d'adaptation au changement climatique.

Dans ce cadre, 116 communes rurales, rassemblées au sein du PNR LAT ont expérimenté des actions de sensibilisation à la maîtrise des consommations énergétiques et aux changements climatiques. Ce territoire, engagé à l'origine pour valoriser les richesses paysagères, culturelles et écologiques et offrir à ses 205000 habitants un espace de vie de qualité, s'est saisi de ce dispositif pour se mobiliser autour des enjeux énergétiques et climatiques. Sa politique se réfère à l'ambition « $d u$ «facteur 4 » qui vise la réduction des émissions de gaz à effets de serre, puis se réoriente ensuite vers le partage d'une culture commune sur l'adaptation locale. Elle vise, à présent, le transfert de ses connaissances et méthodes à ses composantes infra-territoriales à qui la loi confie l'élaboration des «plans climat ».

\section{Le contexte éducatif en direction de la population}

6 Le PNR LAT coordonne une quinzaine d'acteurs éducatifs qui animent sa politique éducative. En 20 118, ceux-ci ne se sont pas encore approprié cette problématique pour développer des propositions au secteur scolaire et encore moins à l'ensemble de la 
population. Ce sont donc conjointement que les chargées de mission, l'une spécialiste du climat, l'autre de l'éducation ont développé des expérimentations de plusieurs modalités basées sur le partage d'informations sur l'évolution du climat avec des habitants.

7 En 2018, 2847 personnes élus, membres d'associations et personnes d'âges divers, investies sur le sujet ou découvrant la problématique, ont participé à des temps de sensibilisation, de débats autour de ces questions ou à travers des spectacles, des ateliers théâtralisés, ou d'ateliers prospectifs organisés par le Parc naturel régional (PNR) dont il est question ici.

Dans cet article, l'analyse met la focale sur les ateliers prospectifs sur les changements climatiques. Ils ont été conçus sur une philosophie de l'action des PNR fondée sur l'accompagnement des acteurs du territoire aux cultures et intérêts parfois divergents, pour élaborer des priorités d'action et une vision d'avenir qui fasse sens pour tous les habitants de ce territoire. Ils conjuguent la mémoire des individus et l'imagination collective de futurs possibles au regard des enjeux écologiques et des trajectoires probables, connues, incertaines, inacceptables... liées aux changements climatiques. Ils s'appuient sur des scénarios élaborés avec des chercheurs et spécialistes du climat, et sur des récits prospectifs à partir de tendances lourdes globales mais déclinées localement en fonction de plusieurs enjeux: santé, ressources en eau, biodiversité, risques naturels, économie locale (l'agriculture, le tourisme, la forêt). La posture méthodologique vise à identifier, faire émerger, valoriser, mettre en œuvre les savoirs citoyens et à faciliter la diffusion et la médiation des connaissances scientifiques dans une logique de co-construction avec les personnes concernées d'orientations et d'actions partagées.

\section{Dialogue réflexif sur l'expérience de médiation climatique}

9 Cet article est le fruit d'un dialogue réflexif entre la chargée de mission " éducation » du PNR LAT médiatrice sur les ateliers et une chercheuse en éducation membre du Conseil scientifique de ce PNR. Les questions initiales portaient sur l'évaluation du dispositif mobilisé en vue de capter l'intérêt et de faire s'exprimer les habitants sur leur appréhension des changements climatiques sur leur territoire de vie.

10 L'analyse s'appuie sur la chronique professionnelle rédigée, à postériori, par la chargée de mission sur son expérience antérieure de différentes médiations sur le climat avec un public adulte souvent appréhendé de manière ponctuelle et individuelle lors de diffusions de spectacles ou d'évènements.

11 Elle est complétée ensuite par l'exploration commune du design pédagogique des " ateliers de prospective territoriale sur le climat " (déroulé, objectifs, support, outils de médiation...), et enfin par l'observation-participante de la chercheuse lors de trois des ateliers. À chaque séance, le dialogue interprétatif s'est construit à partir des plusieurs éléments: les observations à partir d'une grille (notions climatiques abordées, échelles de territoire, thématiques des enjeux prioritaires...), du décryptage des enregistrements des échanges lors des séances, et des questionnements de la médiatrice. Ce dialogue a porté particulièrement sur les significations des interactions médiateur/participants, sur les processus cognitifs liés aux supports proposés, sur les tensions entre les savoirs d'expériences des participants et les connaissances actuelles sur les évolutions locales du climat. 
Dans le cours de cette réflexion, des éléments critiques ont apporté des ajustements de la pratique et des outils et conduit à une expérimentation active lors d'ateliers postérieurs dans un cycle: observation réfléchie, capitalisation, nouvelle expérimentation... Enfin, les lectures partagées d'articles de recherche et l'écriture en commun de cet article finalisant le processus réflexif sont venues renforcer la situation d'énonciation et d'explicitation de la pratique et la conscientisation de l'agir professionnel, de son contexte et sa critique (Kolb, 1984, Schön 1993). L'analyse est complétée par une enquête en ligne à postériori auprès de l'ensemble des participants sur les effets des ateliers.

\section{Production de connaissances expertes sur le climat local et expérimentations de leur diffusion sur le territoire du Parc naturel régional Loire-Anjou-Touraine}

13 La circulation du savoir scientifique ne suffit apparemment pas pour initier un passage à l'acte politique ou citoyen (Marshall, 2017). Deux modèles d'intervention sur les questions scientifiques auprès d'adultes, visant l'engagement et/ou la citoyenneté scientifique, sont en tension: le modèle transmissif visant des adultes considérés comme en déficit de connaissances scientifiques les conduisant à adhérer à des discours irrationnels (Girault, Molinatti 2011, p. 160) et un modèle «dialogal » engageant un « citoyen scientifique » doté de droits et de devoirs, basé sur un communication entre tous les "savoirs" pour utiliser au mieux les formes participatives de débat qui sont essentielles à une démocratie et à une meilleure intégration de la science dans la société (Chavot et Masseran, 2010, p. 2). Quelles sont les différentes postures adoptées au sein de ce PNR?

\section{La production de connaissances localisées fondée sur l'expertise}

Lorsque le PNR LAT s'engage sur les questions climatiques, les problématiques sont loin d'être consensuelles pour la population, y compris au sein des élus des collectivités locales qui siègent dans son Comité syndical. Le défi pour la chargée de mission " climat » est à la fois de former ses collègues et de peser pour que ces questions arrivent à l'agenda des élus. Elle s'attelle à produire avec le secteur de la recherche des données locales pouvant orienter concrètement les actions du PNR. Celle-ci possède une culture scientifique avancée sur les questions climatiques et énergétiques.

Pour décliner les données générales sur le climat, elle a produit des documents de vulgarisation tel "Saumurois, chinonais le climat change et vous?» sur les évolutions climatiques passées 1947-1980 et 1981-2010 et à venir 2021-2050, 2071-2100. Certains éléments sont des données issues d'observations de Météo-France: inventaire des vagues de chaleur, décalage des saisons de 1955 à 1984 et de 1985 à 2014, déficit hydrique entre 1971 et 2000, évolution des précipitations. D'autres ressources cartographiques ont été mobilisées : risque de retrait ou gonflement des argiles, risques "inondation et cavités", issues des Plans de prévention des risques d'inondation (PPRI), schéma national du potentiel de migration des espèces. D'autres repères fournis sont des prévisions «probables » ou «très probables » pour 2050. Les précipitations estivales sont indiquées « variable selon les modèles ». Les conséquences en sont tirées 
sur l'évolution de la Loire et de ses affluents, la biodiversité, les conditions pédoclimatiques (données du Centre Régional de Production forestière), l'agriculture évolution des rendements, dates de récoltes, aires favorables pour la culture de la vigne (données de l'Institut national de recherche agronomique INRA), la santé, les pathologies, la dégradation de l'air et de l'eau.

Le Conseil Scientifique et Prospectif du PNR LAT composé de 22 chercheurs d'écoles supérieures et universités de Tours et Angers est destinataire de ces données et mobilise son expertise et ses ressources universitaires issus des travaux étudiants (Richard, 2013) pour co-produire de la connaissance. La réflexion s'organise à travers des séminaires de deux jours conjuguant des rencontres avec des acteurs d'un microterritoire du PNR et la contribution des savoirs interdisciplinaires en son sein, comme cela a été le cas en 2018 sur les concepts de « résilience » et "d'habitabilité ».

Pour les autres chargés de mission du PNR, organisés dans une logique sectorielle (agriculture/alimentation, habitat/urbanisme, biodiversité), l'appréhension des enjeux climatiques est variable et la "climatisation » (Aykut, 2017) des actions n'est pas mise au centre de leurs missions.

\section{Sensibilisation des habitants à partir de données locales et de leurs savoirs expérientiels}

Depuis 2011, des ateliers sont conduits par le PNR dans une visée d'éducation des adultes. Lors de ces ateliers d'une heure trente à deux heures, les participants sont invités à imaginer la vie, les difficultés, les solutions trouvées par les habitants de 2050. Ces ateliers sont centrés sur la transmission de connaissances localisées sur les changements climatiques, intégrant progressivement les informations plus précises, issues de la recherche, sur l'évolution du climat. Il s'agit d'accompagner les participants pour qu'ils réfléchissent sur des évolutions concrètes de leur lieu de vie en liaison avec les changements climatiques et de les inviter à proposer des adaptations.

Simultanément est diffusé un spectacle "Atmosphère, atmosphère » de la compagnie Spectabilis ${ }^{1}$, co-produit par le PNR LAT, qui circule sur l'ensemble du territoire et audelà. Cette compagnie privilégie les approches créatives basées sur le recueil de témoignages croisés avec la collecte d'informations scientifiques. Après une phase immersive dans la thématique, la création prend forme lors des résidences sur le territoire pendant lesquelles les auteurs, metteurs en scène et comédiens tissent la trame théâtrale en se nourrissant des échanges avec les habitants.

\section{Les ateliers prospectifs sur le climat : une invitation à se projeter en} 2070

20 Le projet d'éduquer les citoyens à l'atténuation et à l'adaptation aux changements climatiques est l'un des sujets les plus délicats auquel les domaines de l'éducation et de la communication sont confrontés (Pruneau, Demers, Khattabi, 2008). Cela implique que les gens se préoccupent mentalement du problème et acquièrent une compréhension de celui-ci, éprouvent une réponse émotionnelle tels l'intérêt, le souci ou l'inquiétude et réagissent activement par des changements de comportement, de mode de vie ou d'action politique liée au climat. 
21 A ces formes ponctuelles de diffusion de l'information succède la proposition faite à des groupes constitués de participer ensemble à un atelier de réflexion sur les évolutions climatiques locales. «L'atelier de prospective territorial sur le climat» se met en place en 2017 et se déroule ainsi. L'atelier commence ! 5 à 10 participants sont invités à embarquer dans une " machine à voyager dans le temps ». Pour préparer leur voyage, ils ouvrent une boîte noire qui consigne des indices de changements sur la période qui sera traversée. Les participants sortent un à un de la boîte les objets qu'elle contient: une photographie d'usines dégageant du $\mathrm{CO} 2$, un thermomètre, une bouteille d'eau presque vide, un parapluie cassé, une image d'hirondelles. Le participant qui choisit un objet propose au groupe une explication à sa présence en mobilisant ses connaissances sur le sujet : hausse des températures liées à l'émission des gaz à effet de serre, sécheresse et étiage des rivières, fragilité de la biodiversité...

Des informations complémentaires sur les impacts potentiels du climat sur le territoire et au-delà sont présentées au fil de la discussion, pour répondre aux interrogations des participants, les inviter à approfondir certains points. Des cartes, des graphiques et des éléments-clés appuient les explications apportées, questionnent ou renforcent les propositions du groupe.

Mieux informé, le groupe est ensuite invité à projeter sur une carte de leur commune, l'avenir du territoire confronté à ces changements et à dialoguer sur les hypothèses d'actions à mener. En fin de discussion, un scénario d'ensemble peut se profiler, fondé sur des évènements-clés qui font figure de points de basculement. L'avenir qui se dessine dans les projections des habitants est fait de multiples pistes de changements. Leur formulation constitue une étape essentielle de la délibération. Les scénarios sont négociés sur de grandes cartes sur lesquelles chacun projette la configuration spatiale de ses propositions.

Enfin pour conclure l'atelier, les participants rédigent une «carte postale » venue du futur à transmettre aux habitants dégageant les enjeux prioritaires. Les propositions sont par la suite mises en image par une graphiste sous forme d'une carte postale géante reflétant la proposition du groupe. Ces visuels et ces textes constituent des supports d'expositions pour relancer l'échange avec d'autres publics lors d'évènementiels.
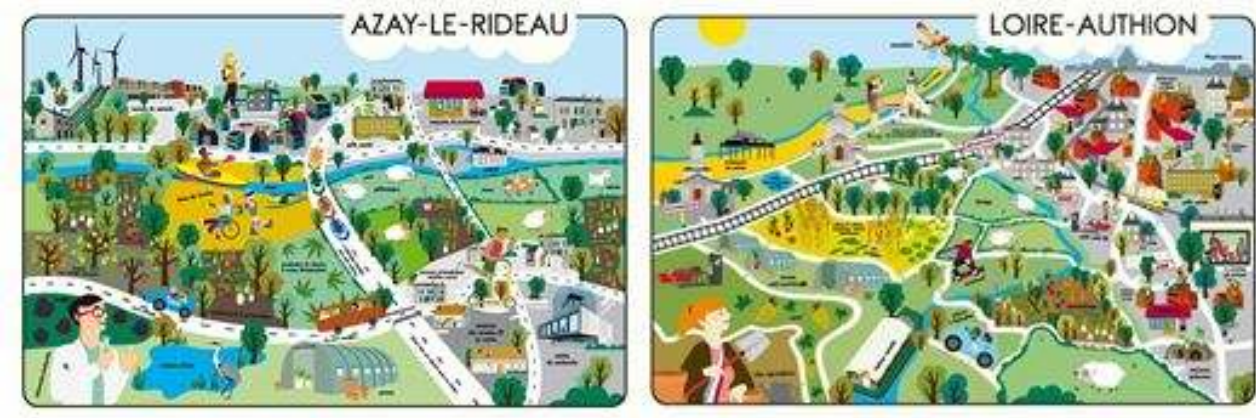

26 Causes, conséquences, solutions? En se centrant sur les effets prévisibles du changement climatique la visée immédiate n'est pas de promouvoir des comportements individuels sobres en carbone. L'atelier cherche principalement à diffuser les informations produites par le PNR LAT et ses partenaires sur les évolutions probables du climat dans le territoire et à mobiliser la population pour concevoir des mesures 
adaptatives, en contribuant «à remettre la question du long terme au cœur de la question publique» (Bourg, 2018, p. 19). L'objectif pour la chargée de mission est d'identifier des orientations portées par la population que le PNR pourrait relayer soutenir ou impulser.

Le tableau suivant présente le processus en quatre étapes : les trois premières étapes se déroulent lors de l'atelier. La dernière étape a été évaluée par enquête à la suite des ateliers.

Tableau 1 : Étapes du processus

\begin{tabular}{|c|c|}
\hline $\begin{array}{l}\text { tion } \\
\text { tion des participants et de leur } \\
\text { ie } \\
\mathrm{n} \text { au voyage et découverte des } \\
\text { e changement climatique } \\
\text { des représentations et } \\
\text { ances initiales du public } \\
\text { d'information, GES, pluies, } \\
\text { ures, migrations des animaux, } \\
\text { en eau }\end{array}$ & $\begin{array}{l}2 \text { Extériorisation } \\
\text { ○ Observations individuelles des } \\
\text { changements (saisons, évènements } \\
\text { météo) } \\
\text { ○ Réactions aux cartes/ graphiques/ } \\
\text { schémas/effets potentiels sur le } \\
\text { territoire } \\
\text { ○ Premières propositions d'actions } \\
\text { O Questionnement réciproque des } \\
\text { participants }\end{array}$ \\
\hline $\begin{array}{l}\text { connaissance des } \\
\text { es } \\
\text { aux enfants, aux proches, à } \\
\text { ge »: collègues, viticulteurs, } \\
\text { mise en monde de problèmes } \\
\text { sés } \\
\text { incertain de prise possible }\end{array}$ & $\begin{array}{l}\text { ison - délibération } \\
\text { entre participants : apport sur } \\
\text { portunités naturels et } \\
\text { ques du territoire } \\
\text { isation avec l'incertitude } \\
\text { tion des propositions sur carte } \\
\text { tion de scénarios } \\
\text { ion d'une «carte postale» } \\
\text { aux habitants d'aujourd'hui }\end{array}$ \\
\hline
\end{tabular}

\section{Les apprenants adultes en petits groupes d'interconnaissance}

276 personnes ont participé à cet atelier, dont $62 \%$ de femmes / 38 \% d'hommes et $60 \%$ d'actifs / $40 \%$ de retraités, $16 \%$ de moins de 30 ans. Ce sont des élus de communes du PNR LAT, des enseignants d'un lycée, des groupes non formels d'habitants, les membres de deux associations culturelles locales, des jeunes en "service civique » et 11 groupes de bénévoles engagés comme "ambassadeurs» auprès du PNR pour assurer la promotion de son projet et de ses actions.

Les ateliers ont donc été menés avec une diversité de groupes, chacun d'entre eux étant composé de personnes aux profils similaires, souvent en interconnaissance. Il a été plus aisé de prendre en compte les individus, leur langage et leurs aspirations grâce à l'homogénéité relative au sein des groupes. Le niveau de motivation de ces participants vis-à-vis des enjeux climatiques est très divers, marqué par la présence conjointe ou non d'une sensibilité écologique et d'une implication dans la vie locale. L'implication citoyenne non accompagnée d'une conscientisation environnementale avérée engendre des scénarios reflétant une capacité à mobiliser une connaissance des enjeux actuels du lieu de vie pour penser son évolution mais peinant à être reliée aux enjeux climatiques. A contrario, les jeunes adultes qui affichaient un souhait d'engagement pour le climat, 
manquaient d'ancrage local et d'assurance pour agir localement sans être soutenu ou devancé par la société. Les élus ou les associatifs qui combinaient ces deux facteurs étaient aptes à scénariser des propositions en mobilisant une pensée systémique. Enfin, les ateliers qui rassemblaient une plus grande diversité de profils produisaient des niveaux de propositions assez disparates, peu scénarisées et aboutissant rarement à une mise en système des idées. Dans tous ces cas de figure, ces ateliers étaient souvent pour les participants l'occasion de remobiliser et de partager des solutions concrètes observées dans d'autres contextes.

Dans tous ces groupes, il a été possible de repérer des individus «branchés » (Moser, et Dilling, 2007) qui possèdent un vaste réseau de contacts et sont à même de diffuser l'information (élus, associations culturelles, jeunes adultes) et quelques rares " érudits » déjà impliqués et aptes à trouver et à mettre en œuvre les réponses aux questions (par leur activité professionnelle le plus souvent).

\section{Présentation des résultats et discussion}

31 L'analyse des ateliers a mise en évidence trois points saillants. Le premier permet de caractériser les interventions de la chargée de mission entre médiation narrative sur l'avenir du territoire et sollicitation des savoirs locaux des participants. Le deuxième point porte sur la forte dimension émotionnelle de la question du climat. Enfin, le troisième met en évidence d'autres "épreuves " du territoire avec les risques d'inondation et d'accident nucléaire.

\section{Positionnement de l'activité de médiation : co-élaboration centré sur le local}

32 Le changement climatique est une menace existentielle d'une ampleur inégalée. Nous participons tous activement à le négliger en élaborant des récits individuels qui nous permettent de gérer l'angoisse, de différer les changements que nous devrions faire, en le rendant plus distant, moins certain, plus désespéré. Il est menaçant pour nos identités, notre habitat, notre mode de vie, nos attentes pour l'avenir et celui de nos enfants. Le projet de l'atelier est de participer à la construction des savoirs pour et par les individus dans de petits groupes facilitant le partage des observations et expériences localisées.

Les interventions de la "médiatrice» combinent la posture scientifique (concepts, discours sur les incertitudes), la position d'expertise (en abordant les risques, la vulnérabilité) ou politique (le principe de précaution). Elle signale ses ignorances lorsque ces sujets sont abordés par les participants (effets du réchauffement climatique sur les cavités des troglodytes, remontée des marées au-delà de l'estuaire de la Loire).

Elle s'efforce de privilégier la posture dialogique d'un entrelacement de voix différentes couplées avec la diffusion prudente des données, en tension entre déontologie scientifique - s'en tenir aux connaissances - et éthique conséquentialiste - alerter sur les risques. Lors des ateliers observés, elle communique sur ces deux plans en se gardant d'être catastrophiste ou normative. Elle cherche essentiellement à favoriser l'expression des participants sur leurs connaissances et leurs visions de l'avenir climatique. Elle adopte - au nom du PNR - une position de médiation narrative des 
changements potentiels du territoire. Elle recherche la création d'un climat de confiance avec le groupe pour favoriser une expression sécurisée, guidée par une reformulation empathique qui permet d'avoir accès à soi et aux autres (Sirota, 2020, p. 36).

Si le public fait souvent référence aux manifestations globales des changements climatiques : sécheresses, incendies, inondations, cyclone, vulnérabilité des côtes, il est encore peu au fait des impacts locaux. Il observe des changements : débuts de floraisons avancées, gels tardifs préjudiciables à la viticulture, basses eaux et sables visibles en Loire. Pour F. Bertrand, ces observations ont une valeur réelle complémentaire aux données scientifiques. "La variété des signes reliés au changement climatique, les savoirs locaux élargissent le champ du problème ». La territorialisation du changement climatique n'est pas seulement une affaire de "descente d'échelles » c'est-à-dire de passer d'une échelle globale à une échelle locale mais aussi un élargissement du problème à un grand nombre de sujets. "Ce sont une multitude d'indications de modifications de l'environnement qui prennent sens dès que les gens s'attardent sur ce qui change autour d'eux» (Bertrand, Alii, 2017). Ces savoirs locaux sont sollicités et intégrés aux données partagées, mais un hiver rigoureux et un printemps très pluvieux peuvent venir perturber les représentations et créer de la confusion. Les supports préparés viennent alors redonner les grandes tendances: température plus élevées l'hiver et canicules et sécheresses plus fréquentes, sols argileux instables, incertitudes que les pluies, orages et tempêtes plus présents, inondation plus fortes sur les petits cours d'eau, diminution de la ressource en eau disponible, et diversification des insectes, virus et parasites, évolution de la répartition des espèces.

Certains phénomènes sont invisibles ou méconnus. La dynamique des facteurs perturbateurs et la manifestation des impacts ne s'inscrivent pas dans la même temporalité, ce qui complique grandement l'appropriation de données qui restent assez générales.

\section{Prise de connaissance, expression de l'inquiétude}

La conscience de ces dérèglements crée une angoisse diffuse sans nécessairement que soient compris les phénomènes d'accélération, les notions de seuils, de rétroactions amplificatrices, les effets de retardement menant à des « points de basculement ».

38 Animer ce type d'atelier met à rude épreuve au plan psychologique et moral et nécessite de comprendre ses propres stratégies de défense contre l'anxiété, repérer la perplexité du public ou son désespoir et ajuster ses supports, son argumentaire, ou rechercher une plus grande précision des informations. L'expression de cette anxiété se traduit par des manifestations physiques (postures d'affaissement, soupirs) voir des appels à l'aide verbaux observées et recueillis par la médiatrice mais n'ayant pas fait l'objet d'une analyse spécifique.

La dimension émotionnelle au plan individuel n'est pas prise comme élément central de l'approche des ateliers du PNR LAT, comme c'est le cas par exemple dans les "Conversations carbone » testées dans quelques sites en France qui s'appuient sur les analyses de Ro Randall (Randall, 2015). Le cœur de ce dispositif de six ateliers des "Conversations carbone » consistent en effet à amener les individus à se connecter à leurs émotions et valeurs, en lien avec le réchauffement climatique et à exprimer la 
signification émotionnelle que revêt le fait de s'engager dans des changements profonds (La Revue Durable, 2016).

40 L'enquête a postériori sur les effets des ateliers affine la compréhension de la composante émotionnelle. Une participante dit avoir été marquée par les projections sur l'élévation importante de la température, le manque d'eau. "J'ai été à la fois impressionnée et attristée, même si j'avais déjà conscience des modifications du climat, mais pas à ce point ». Un autre participant écrit qu'il «ne savait pas vraiment, ne connaissait pas les pronostics sur les effets $d u$ changement climatique et les variations attendues dans la région. Il a ressenti « de l'inquiétude » et "se sent plus concerné qu'avant ». Un habitant a " ressenti de la gêne, car on sait que cela peut être sinon évité, au moins atténué et retardé». Il se dit conscient de ces bouleversements et souligne le "véritable échange mais aussi une inquiétude". L'inquiétude s'accompagne chez l'un des contributeurs d'un sentiment d'impuissance et exprime une faible confiance dans son pouvoir d'agir: "c'est alarmant mais individuellement, que pouvons-nous faire? Les mesures à prendre doivent être collectives et initiées par nos hommes politiques ». Un seul témoignage manifeste une évolution vers plus d'optimisme en découvrant que les dommages seront moindres que dans d'autres pays du monde : "J'étais pessimiste, maisj'en suis sorti un peu plus optimiste...

41 Ces expressions témoignent de la diversité des niveaux d'appropriation des enjeux liés au changement climatique. L'atelier intervient à un moment différent dans la conscientisation des participants. Pour certains, il révèle une angoisse nouvelle ou déniée, d'autres se heurtent aux modalités possibles de passage à l'action et aux échelles de décision, enfin pour certains participants l'atelier permet d'envisager un dépassement de l'inquiétude vers des actions concrètes. L'enjeu de l'atelier est de favoriser, à un moment donné, le franchissement des barrières psychologiques qui bloquent le message climatique «en proposant un récit dramatique qui n'écrase pas tous les possibles» (Hache, 2014). Il faut des petits groupes pour cela conduits par des personnes équipées pour susciter un climat de confiance adéquat qui permet à chaque participant d'être là et d'avoir accès à soi et aux autres, présentement, ici et maintenant tout en conscientisant des mises en crises à venir, de grande envergure, inédite et hyper complexes.

\section{L'inondation et le nucléaire plus angoissant que climat !}

«Quand l'environnement vacille, la vulnérabilité psychique apparaît » (Sirota, 2020). Le travail groupal de médiation reste largement à explorer. Et chacun, habitant comme médiateur a besoin de lieux, de temps pour mettre en forme ses savoirs, identifier ce que ses défenses ont fabriqué, augmenter son territoire psychique de manière féconde et chercher à apprendre de l'inadvenu. Il faut pour cela des médiateurs qui «savent entretenir en eux une porosité et une disponibilité psychiques adéquates pour recevoir et dire en restituant à un autrui, tout en s'adressant attentivement à tout un groupe, quelque chose de ce qu'ils ont cru comprendre de ce qui a été énoncé dans cette situation » (Sirota, 2020, p. 38).

Lors des ateliers observés en 2018, la charge émotionnelle potentiellement générée par les prises de conscience des effets du changement climatique est latente. L'expression de l'angoisse sur l'avenir se détourne sur deux autres sujets majeurs localement: les inondations dévastatrices et le nucléaire. 
Ainsi un groupe, dans son scénario d'avenir, se focalise sur une "épreuve » : celle d'une inondation massive dans la vallée de la Loire angevine. Ce risque est très prégnant sur le territoire, mais il n'est pas attendu que le changement climatique ait un impact qui le renforce. C'est pourtant le scénario d'avenir que choisit de traiter ce groupe composé de plusieurs élus locaux et qui fait l'hypothèse qu'une crue centennale a détruit une grande partie du bâti de la vallée en " 2036 ». Cet évènement attendu et dramatique a permis au territoire de changer de manière radicale de trajectoire.

L'accident nucléaire est un autre motif de manifestation d'angoisse. Il est exprimé dans les groupes souvent par plusieurs participants. Il peut être interprété comme la manifestation d'un biais cognitif : pourquoi se préoccuper du changement climatique alors que la plus grave menace que nous subissons localement est celle du nucléaire, tant dans le risque accidentel que dans les conséquences économiques de son arrêt potentiel.

De fait, le changement climatique relance la question du nucléaire sur le territoire. Les participants prennent conscience de problématiques nouvelles avec le fleuve à l'étiage, au débit réduit et avec des températures de l'eau en hausse. Cette question devient un élément important dans la perspective annoncée d'une pénurie d'eau. EDF pourrait se voir contrainte de réduire la puissance de certains réacteurs ou de les arrêter. Elle modifie les problèmes posés par la sécurité nucléaire et renforce les risques de dégradation de la qualité de l'eau avec leurs conséquences sur les écosystèmes et indirectement sur le tourisme (baignade, paysages). Enfin, elle pose la question cruciale et potentiellement conflictuelle du partage de la ressource en eau et de la priorisation de ses usages.

Depuis ces ateliers, la question des changements climatiques est devenue une préoccupation centrale, présente au cœur des débats politiques et sociétaux. Les effets $\mathrm{du}$ changement climatique (sécheresse, fortes températures, dérèglements) s'invitent aujourd'hui dans la vie quotidienne de chacun et favorisent son appréhension plus concrète.

Les participants ont intégré dans leurs représentations la notion du risque lié aux crues et à l'énergie nucléaire autant par la transmission des connaissances locales que par leurs expériences personnelles. Cependant, ils ne maitrisent pas les données systémiques nécessaires à l'appréhension du risque complexe que représente le changement climatique sur les risques déjà connus (Lammel, 2015).

\section{Construire des scénarios d'avenir : la participation des habitants à un territoire apprenant}

49 En proposant une projection sur 2070, les ateliers mettent en jeu une dramatisation qui implique rétrospectivement la mobilisation d'une intention d'agir dans le présent. En identifiant des leviers pour agir, ils soulignent également des points aveugles et les démarches à poursuivre pour devenir un « territoire apprenant ».

\section{Prendre conscience que l'avenir est déjà là}

L'atelier invite à faire le récit d'un territoire situé dans un futur assez lointain pour envisager des aléas successifs, potentiellement des chocs et imaginer des phases 
d'ajustements, des adaptations expérimentées. L'intégration de nouvelles connaissances sur nos vulnérabilités se conjugue paradoxalement au présent en examinant les normes techniques de nos habitats (ilots de chaleurs urbain), les ressources agricoles plus localisées pour notre alimentation (moins carnée), notre santé (allergies, pollution de l'eau).

51 La « ruse prospective " déjoue nos systèmes de défense et d'évitement et nos biais cognitifs habiles à renvoyer à l'ailleurs ou aux générations lointaines les responsabilités qui nous incombent.

52 Le rôle de la prospective n'est évidemment pas de prévoir l'inattendu, mais d'explorer les voies de l'avenir et de mieux nous armer à faire face, le cas échéant, à des crises provoquées par l'inattendu (Papon, 2016, p. 37) Invité à se projeter dans l'avenir, intérieurement, chacun calcule: quel âge j'aurai en 2070 ? Je serai mort? Et mes enfants? Quel âge auront mes petits enfants ? Est-ce à dire que nous avons jusque-là avant de vivre les effets du changement ? Ce n'est pas le cas, mais faut-il se projeter sur les catastrophes à venir pour éclairer ce qu'il faut entreprendre aujourd'hui ? Peut-on penser l'avenir si les effets irréversibles sont avérés? Ces questions sont redoutables car elles nous confrontent à l'impossibilité d'imaginer un futur meilleur. Jean-Pierre Dupuy propose de se servir de la catastrophe, d'envisager les points de basculement, comme éléments de "révélation » pour nous obliger à repenser le destin de l'humanité (Dupuy, 2002). De combien de temps disposons-nous avant d'atteindre des points de rupture (ex : effondrement des calottes glaciaires, relâchement du méthane possible si le $+2^{\circ}$ est atteint) ?

53 Pour les participants, les données 2070 sur les températures, les prévisions de quatrevingt dix jours de canicule annuelle, les perspectives d'un manque d'eau crucial sont des éléments de dramatisation impliquant rétrospectivement la nécessité d'agir aujourd'hui.

\section{Des scénarii anthropocentrés}

54 Les groupes constituent des petites communautés ponctuelles et créent un espace d'apprentissage sûr, pour explorer, modéliser, tester dans une atmosphère encourageante et plus informée. La concertation et la mise en commun des compétences de chacun dans une projection partagée contribue à sensibiliser les participants aux changements générateurs de comportements sobres en carbone, et renforce chez la plupart d'entre eux leur confiance dans la possibilité de changements dès à présent.

55 En nous rendant plus concernés par la situation, les problèmes environnementaux s'inscrivent au cœur de nos relations intimes avec ce que l'on mange, la manière dont on se déplace, s'habille, se loge, et dont cohabite avec la « nature ».

56 La synergie de chaque groupe de participants, dans le temps bref de l'atelier, met en mouvement les propositions pour le territoire. Chaque atelier montre un aspect de la manière dont les habitants de manière empirique prennent en compte les aspects physiques et matériels des changements. Les thèmes de la consommation, de l'alimentation et de l'agriculture, de l'habitat et de l'énergie, de la mobilité et des transports sont toujours abordés à des degrés divers. 

s'approprier une diversité d'innovations souvent transférée de modèles urbains (tramway, piste cyclable, écoquartier, densification de l'habitat, travail à distance). Sans réelle originalité dans ce court temps de réflexion, le dialogue et l'échange des propositions contribuent à faire monter l'acceptabilité sociale de ces solutions. Penser l'existence de mondes alternatifs, c'est bien sûr une question de valeur, une question de définition de ce à quoi nous sommes attachés pour construire un monde commun, une définition de l'ordre social que nous souhaitons voir advenir.

Si l'évolution des pratiques agricoles est soulevée, la description de ces cultures à venir est plus délicate à traiter. Les incertitudes dans ce domaine étant importantes: les variétés actuelles seront-elles modifiées, d'autres seront-elles introduites, transformées à leur tour pour s'adapter à des conditions climatiques particulières? Cette question intéresse particulièrement le territoire étant donné l'importance du pôle semencier en Maine-et-Loire et le poids de la viticulture.

Qu'est-ce que les récits laissent de côté ? Des points communs peuvent être relevés : les réflexions sont centrées sur le territoire de vie et envisagent rarement les solidarités extraterritoriales. Elles concernent essentiellement les humains et portent peu (pas) sur les «non-humains ». L'évolution des espèces n'est pas appréhendée (migration ou disparition des espèces), et les participants ne manifestent pas de préoccupation majeure sur ces questions. Les connaissances relatives à la biodiversité et à sa vulnérabilité sont faibles. Le végétal n'est pas considéré comme un patrimoine. Spontanément son rôle comme atténuateur des effets du réchauffement est valorisé, sans pour autant que les participants l'identifient concrètement : évapotranspiration, stockage $\mathrm{du} \mathrm{CO} 2$, filtration des particules polluantes, régénération des nappes phréatiques, limitation de l'érosion des sols.

\section{Vers un territoire « apprenant » : décrire le monde et pluraliser les récits}

60 Réunir ainsi des habitants, les connecter avec les ressources en informations, actions possibles, réflexions partagées leur procure par le collectif un pouvoir qu'ils n'ont pas à titre individuel. Cela passe dit Latour par la « description du monde » pour que les gens reprennent une position politique. Passer de la peur à la curiosité, à la découverte, apprendre que la question de l'eau concerne tout un bassin versant, réoriente potentiellement la gestion agricole, qu'elle impacte le maintien du centre de production nucléaire et que la pénurie d'une eau de qualité nécessite dès à présent d'anticiper pour hiérarchiser les priorités.

61 Pour Eric Favey (2018), un « territoire apprenant » apparait à la fois comme l'espace où travaillent et coopèrent des «apprenant/sachant » mais aussi un territoire qui nous enseigne, nous transmet, nous apprend. La compréhension, la participation l'adhésion et l'engagement des populations elles-mêmes constituent un enjeu majeur de démocratie autant que d'efficacité. Le territoire apprenant est le lieu qui permet à l'individu d'aller vers la sphère publique (Caron, 2018, p 32). Le changement climatique ne peut s'envisager comme une série de problèmes auxquels il faudrait apporter des réponses mais comme une dynamique nécessitant une appréciation et une appropriation constamment renouvelée du devenir collectif.

Éducation relative à l'environnement, Volume 16 - 1 | 2021 
62 Les démarches à entreprendre dépendent d'initiatives diversifiées, où le politique a toute sa part, qui n'ont pas de caractère strictement mécanique. Elles nécessitent pour être mises en œuvre des accords collectifs larges relevant de processus sociaux délicats et compliqués (Charles, 2006, p.51). Sur le territoire étendu du PNR LAT, les participants perçoivent la nécessité de lieux de débat et d'arbitrage des priorités.

63 L'avenir qui se dessine apparaît, à court et sans doute aussi à moyen terme, marqué par une très grande confusion, dans laquelle il sera très important mais également aussi très difficile de saisir clairement des perspectives d'action véritablement cohérentes. Cette confusion est une composante incontournable du problème. La multiplicité des domaines à aborder peut donner le vertige. Le défi majeur est probablement d'inscrire toute initiative dans un très fort contexte d'incertitude intégrant les dimensions globales aux stratégies locales.

\section{Conclusion}

En centrant son projet sur l'élaboration par les habitants d'une vision générale de leur vie future, ces ateliers contribuent à l'appropriation des données localisées sur les effets des changements climatiques. Les participants, tout en manifestant leur inquiétude, leur gène devant l'inaction, développent des projections dans des vies futures satisfaisantes en y intégrant à travers leur dialogue des propositions qui vont bien au delà des "petits gestes quotidiens". Ils découvrent les impacts sur les écosystèmes mais leurs connaissances et leur décentrement dans l'approche de la biodiversité reste limités.

Ils s'initient à la mise en lien des composantes du territoire qui vont être impactés et aux échelles de temps et d'incertitudes qui n'évacuent pas la nécessaire anticipation. $\mathrm{Au}$ contraire, la projection dans le futur rend plus valide la nécessité de changements dès aujourd'hui contribuant à l'atténuation et préfigurant les adaptations et les étapes à négocier.

Le territoire « éducatif » ne devient pas nécessairement un territoire " apprenant » s’il en reste à l'idée de changements individuels, sans exploration du territoire comme bien commun. L'intégration de ces connaissances d'ordre cognitif, stratégique et affectif est cruciale pour que se construise un savoir agir politique : savoir dénoncer, résister, choisir, proposer, créer... (Sauvé, 2017). Il nécessite pour les PNR et les acteurs locaux, une mise en chantier permanent dont les modalités méthodologies restent grandement à inventer.

67 Ces ateliers ne sont qu'un élément modeste d'entrée dans des processus d'adaptation continue et dans la construction d'une culture partagée de l'adaptation. Ils indiquent l'intérêt de créer des collectifs et des récits de territoire pour nous accompagner dans la terrible aventure de la lutte contre l'effondrement (Diamond, 2006) sans se laisser submerger par le risque de nous effondrer nous-mêmes et sans possibilité d'imputer les situations de menaces à des causes externes. Notre capacité à évoluer vers une meilleure réalité sociale dépend essentiellement de notre capacité à nous attaquer à notre désespoir. L'espoir doit être ancré dans la pratique par l'action déterminée et s'actualiser dans la question : qu'allons-nous faire? 


\section{BIBLIOGRAPHIE}

Aykut Stefan Cihan., (2017). La « gouvernance incantatoire ». L'accord de Paris et les nouvelles formes de gouvernance globale. lapenseeecologique.com. Points de vue. 1 (1). Consulté sur http:// lapenseeecologique.com/la-gouvernance-incantatoire-laccord-de-paris-et-les-nouvelles-formesde-gouvernance-globale/

Bertrand, F. et Richard, E. (2014). L'action des collectivités territoriales face au « problème climat » en France : une caractérisation par les politiques environnementales. Natures sciences sociétés, 22(3) p 195-203.

Bertrand, F., Petit, S., Vergote, M.-H. et Brayer, J.-M., (2017). Design territorial et changement climatique : innover pour s'adapter à une ressource en eau incertaine. Innovations 54(3), p. 41-63.

Bonduelle A. et Jouzel, J. (2014). L'adaptation de la France au changement climatique mondial, Rapport au Conseil économique social et environnemental. Paris : Édition des journaux officiels

Bourg, D. (2018). Le citoyen est au cœur de la démocratie comme de l'environnement. Dans L'expérience du territoire, Apprendre dans une société durable (p. 18-21). Diversité $\mathrm{n}^{\circ} 191$

Brelet,M., Novel. A., Olagnie, R., Dupré, M., Martin, V., Derkenne, C., Cazenave, C., Millet, A., (2017), Des images et des actes : quels visuels pour parler du climat, Site : www.desimagesetdesactes.fr Candea, P. (2017). Pour vivre une conversation carbone il n'est pas nécessaire d'être un expert. LaRevueDurable, $\mathrm{n}^{\circ} 60$, p. 64-67

Charles, L. (2006/2). Perspectives sur l'histoire de la météorologie et de la climatologie. Écologie \& politique 33 (2), 36-52

Caron, J-F. (2018). Le territoire apprenant, c'est le lieu qui permet à l'individu d'aller vers la sphère publique. Dans L'expérience du territoire, Apprendre dans une société durable (p. 32-36). Diversité, $\mathrm{n}^{\circ} 191$

Chavot, Ph., Masseran, A. (2010). Engagement et citoyenneté scientifique : quels enjeux avec quels dispositifs? Questions de communication, 17. Consulté sur http:// questionsdecommunication.revues.org/374

Diamond, J. (2006). Effondrement. Comment les sociétés décident de leur disparition ou de leur survie. Paris : Gallimard

Dupuy, J.-P. (2002). Pour un catastrophisme éclairé. Paris : Éditions du Seuil

Favrey, É. (2018). Apprendre par et pour les territoires. Dans L'expérience du territoire, Apprendre dans une société durable (49-53). Diversité $\mathrm{n}^{\circ} 191$

Girault, Y. et Barthes, A., (2016). Postures épistémologiques et cadres théoriques des principaux courants de l'éducation aux territoires. Éducation relative à l'environnement, 13(2). Consulté sur http://journals.openedition.org/ere/755

Girault, Y. et Molinatti, G. (2011/3). Comment les musées et centres de sciences s'exposent aux controverses socioscientifiques. Hermès, La Revue, 61(3), 159-166.

Hache, E. (dir.) (2014). De l'univers clos au monde infini. Paris : Éditions. Dehors

Kolb, D. A. (1984), Experiential learning experience as a source of learning and developement, New Jersey : Prentice Hall 
Lammel, A. (2015). Changement climatique de la perception à l'action, note $n^{\circ} 5$. Paris : Fondation à l'écologie politique

Latour, B. (2017). Où atterir ? Comment s'orienter en politique. Paris : La découverte

Marshall. G. (2017). Le syndrome de l'autruche. Pourquoi notre cerveau veut ignorer le changement climatique. Arles : Actes sud

Moser, S. et Dilling, L. (eds.) (2007). Creating a climate for change : commnicating climate change and faciliting social change. Cambridge University Press

Obœuf, A. et coll. (2016). Les méthodes de codesign : une ressource capacitante. Journal International de Bioéthique, 27(1), 127-146

Oreskes, N. et Conway M. E. (2012). Les marchands de doute. Paris : Le Pommier

Papon, P. (2016/2). La prospective, de l'anticipation à la préparation de l'avenir. Annales des Mines - Réalités industrielles 2, 33-37

Pruneau D., Demers M. et Khattabi A. (2008). Éduquer et communiquer en matière de changements climatiques : défis et possibilités. Vertigo - la revue électronique en sciences de l'environnement, 8 (2). Consulté sur http://journals.openedition/org/vertigo/4995

Randall R., Brown A. (2015). In time for Tomorow ? The carbon conversation handbook. Striling : The Surefoot effect

Richard, E. (2013). L'action publique territoriale à l'épreuve de l'adaptation aux changements climatiques : un nouveau référentiel pour penser l'aménagement du territoire? Thèse de doctorat en Aménagement de l'espace et Urbanisme, Université de Tours. Consulté sur http:// www.applis.univ-tours.fr/theses/2013/elsa.richard_4160.pdf

Sauvé, L. (2017). Éducation à l'écocitoyenneté. Dans Barthes, A., Lange, J.-M., et Tutiaux-Guillon N., Dictionnaire critique. Des enjeux et concepts des «éducations à » pp. 56-65). Paris : L'Harmattan.

Schön, D. (1994). Le praticien réflexif. À la recherche du savoir caché dans l'agir professionnel (J. Heynemand et D. Gagnon, trad.). Montréal, Canada : Les Éditions Logiques. (Ouvrage original publié en 1983 sous le titre The Reflective Practitioner. New York, NY : Basic Books).

Sirota, A. (2020/8). Quand l'environnement vacille, la vulnérabilité psychique apparaît. Le journal des psychologues, $\mathrm{n}^{\circ} 130,34-38$

\section{NOTES}

1. Voir www.ciespectabilis.com

\section{RÉSUMÉS}

L'article présente les actions conduites par un Parc naturel régional (PNR) français avec des habitants de son territoire pour les sensibiliser aux changements climatiques. La nature même des enjeux climatiques soulève de délicates questions stratégiques en matière de communication 
et d'information de la population. Parmi toutes les démarches menées par le PNR pour la diffusion des données scientifiques élaborées sur les effets locaux des changements climatiques, l'analyse porte spécifiquement sur l'animation «d'ateliers de prospective territoriale » conduits avec des groupes d'habitants pour initier une dynamique collective et identifier les enjeux engageant l'avenir de l'espace géographique du PNR.

La réflexion est issue du dialogue et d'une co-écriture entre une chargée de mission «éducation » et une chercheuse en observation participante sur trois des ateliers. La philosophie des ateliers se fonde sur la projection sur l'avenir articulant savoirs citoyens expérientiels et connaissances individuelles sur le climat, et les savoirs scientifiques et scénarisations contrastées et hypothétiques de tendances sur le territoire afin de construire une vision débattue voire partagée des actions à mener.

Le détour par une projection en 2070, à travers les co-productions des habitants dans ces ateliers, est expérimenté comme « ruse rétrospective » : conscientisant les scénarii de l'inacceptable, pour définir collectivement les enjeux à relever. Il a pour effet pour les participants de se positionner dans le présent comme plus proactif face à l'avenir. L'atelier met en évidence la nécessité de structures institutionnelles propices à la délibération et un territoire « apprenant » permettant durablement aux individus d'aller vers la sphère publique.

The article presents the actions carried out by a French Regional Natural Park (PNR) with inhabitants of its territory to make them aware of climate change. The very nature of climate change raises delicate strategic questions in terms of communication and public information. Among all the proceeding taken by the Park for the dissemination of scientific data developed on the local effects of climate change, the analysis relates specifically to the animation of "territorial prospective workshops" conducted with groups of residents to initiate a collective dynamic and identify the issues involving the future of the PNR geographic area.

The reflection result of a dialogue between an "education" project manager and an observation researcher participating in three of the workshops.

The philosophy of the workshops is based on the projection on the future articulating experiential citizen knowledge and individual knowledge on the climate, and scientific knowledge and contrasting and hypothetical scenarios of trends on the territory, in order to build a discussed, even shared vision of actions to be taken.

The detour through a projection in 2070, through this co-productions of the résidents in these workshops, is experienced as a "retrospective ruse": raising awareness of the scenarios of the unacceptable, to collectively define the issues to be addressed. It has the effect for participants of positioning themselves in the present as more proactive in the future. The workshop highlights the need for institutional structures conducive to deliberation and a "learning" territory that allows individuals to move into the public sphere on a lasting basis.

\section{INDEX}

Mots-clés : éducation des adultes aux changements climatiques, atelier prospectif territorial, participation des habitants

\section{AUTEURS}

\section{DOMINIQUE BACHELART}

Dominique Bachelart est maitre de conférences en sciences de l'éducation de l'Université de Tours, membre du Conseil Scientifique et Prospectif et de la commission éducative du Parc 
naturel régional Loire Anjou Touraine (PNR LAT) Ses travaux de recherche portent sur différentes dimensions de l'éducation au territoire, comme lieu de vie avec le courant de l'écoformation, comme espace stratégique autour d'enjeux agri-environnementaux et sur le « territoire » comme lieu d'appropriation des enjeux environnementaux et d'engagements publics.

\section{EMMANUELLE CRÉPEAU}

Emmanuelle Crépeau est chargée de mission « éducation-participation » depuis 2008 au Parc naturel régional Loire-Anjou-Touraine (PNR LAT). En 2011, elle conçoit des ateliers de prospective territoriale sur le changement climatique en direction des adultes. En 2018-2019, elle pilote la concertation pour la révision de la charte du PNR, élaborée pour quinze ans. En 2020, elle s'engage comme « garante » des procédures de démocratie participative auprès de la Commission Nationale de Débat Publique (CNDP). 